Communications in Physics, Vol. 19, No. 4 (2009), pp. 201-204

\title{
ORBITAL OSCILLATOR COMMUTATION RELATIONS AND MASS SHIFTING FOR SUPERSTRING
}

\author{
NGUYEN THI HA LOAN \\ Hanoi Pedagogical University No. 2 \\ NGUYEN HONG HA \\ Institute of Physics and Electronics VAST
}

\begin{abstract}
In this work we extend the results obtained in [5] on mass shifting for bosonic string to the case of superstring. The modified anomaly terms of superstring superalgebras are shown and the corresponding BRST charge is used.
\end{abstract}

\section{INTRODUCTION}

Superstring theory [1-4] is considered as a prospective direction for the construction of unified theory of all fundamental interactions. On this way there are, however, some difficulties to overcome, among these is the existence of particle with negative squared mass called tachyon.

The authors of Ref. 5 have considered a model of bosonic string, in which the mass spectrum of component fields can be shifted as compared to conventional theory, and hence the tachyon can be automatically removed.

The aim of our work is to extend the result in [5] to the case of superstring. It is show that in this case we can also construct a superalgebra with a modified anomaly term is such a way that the theory does not contain tachyon field without the use of GSO mechanism [6]. The contents of the paper are arranged as follows. In Sec. II we construct the superalgebra with a modified anomaly term in the spirit of Ref. 5. In Sec. III the BRST charge for superstring is treated with a modified anomaly term. Sec. IV is devoted to the equations of motion and the mass spectrum for component fields.

\section{MODIFIED NEVEU-SCHWARZ AND RAMOND SUPERALGEBRAS}

In Ref. 5 on the base of modified commutation relations for orbital oscillators

$$
\begin{aligned}
{\left[\alpha_{n}^{\mu}, \alpha_{m}^{\nu}\right] } & =\left(-n . \eta^{\mu \nu}+G^{\mu \nu}\right) \delta_{n,-m}, \\
{\left[\pi^{\mu}, \pi^{\nu}\right] } & =G^{\mu \nu},\left[P^{\mu}, \pi^{\nu}\right]=0,
\end{aligned}
$$

the Virasoro algebra is derived

$$
\left[L_{n}, L_{m}\right]=(n-m) L_{n+m}+A(n) \delta_{n,-m},
$$

with modified anomaly term

$$
A(n)=\frac{D}{12} n\left(n^{2}-1\right)+\frac{1}{2} n G^{2}
$$


where $G^{\mu \nu}$ is some antisymmetric tensor,

$$
\begin{array}{r}
G^{2} \equiv G_{\mu \nu} G^{\mu \nu} \\
\alpha_{0}^{\mu}=p^{\mu}+\pi^{\mu}, \quad \pi^{\mu}|0\rangle=0
\end{array}
$$

To extend this model to the case of superstring we processed as follows. Put

$$
L_{n}=L_{n}^{(x)}+L_{n}^{(\psi)}
$$

where $L_{n}^{(x)}$ are Virasoro generators related to the coordinate $X_{\mu}$ and satisfy the commutation relations $(2)$ and $(3), L_{n}^{(\psi)}$ are Virasoro generators related to the supercoordinate $\psi$ and satisfy the commutation relations

$$
\left[L_{n}^{(\psi)}, L_{m}^{(\psi)}\right]=(n-m) L_{n+m}^{(\psi)}+A_{n}^{(\psi)} \delta_{n,-m}
$$

In accordance with equations (2) and (3) we consider the case when $A_{n}^{(\psi)}$ has an additional term proportional to $n G^{2}$, namely

$$
A_{(n)}^{(\psi)}=\frac{D}{24} n\left(n^{2}+2-3 \delta\right)+g_{\psi} \cdot n G^{2}
$$

where $g_{\psi}$ is some parameter,

$$
\delta= \begin{cases}1, & N S \text { sector } \\ 0, & R \text { sector }\end{cases}
$$

Now we have

$$
\begin{aligned}
& A(n)=A_{(n)}^{(x)}+A_{(n)}^{(\psi)}=\frac{D}{8} n\left(n^{2}-\delta\right)+g \cdot n G^{2} \\
& g \equiv \frac{1}{2}+g_{\psi}
\end{aligned}
$$

According to (7) we have the superalgebra of the form

$$
\begin{aligned}
& {\left[L_{n}, L_{m}\right]=(n-m) L_{n+m}+\left[\frac{D}{8} n\left(n^{2}-1\right)+g . n G^{2}\right] \delta_{n,-m}} \\
& {\left[L_{n}, G_{r}\right]=\left(\frac{n}{2}-r\right) G_{n+r}} \\
& \left\{G_{r}, G_{s}\right\}=2 L_{n+s}+\left[\frac{D}{2}\left(S^{2}-\frac{\delta}{4}\right)+g G^{2}\right] \delta_{r,-s}
\end{aligned}
$$

\section{BRST CHARGE}

It is known (see e.g [7]) that the expression of BRST charge for superstring is:

$$
\begin{aligned}
Q= & \sum_{n \in Z} L_{n} C_{-n}+\sum_{\lambda} G_{\lambda} \gamma_{-\lambda}+\frac{1}{2} \sum_{n, m \in Z}(n-m): C_{-n} C_{-m} b_{n+m}: \\
& +\sum_{n} \sum_{\lambda}\left(\frac{n}{2}-\lambda\right): \beta_{n+\lambda} \gamma_{-\lambda} C_{-n}:-\sum_{\lambda, \rho}: b_{\lambda+\rho} \gamma_{-\lambda} \gamma_{-\rho}-a_{0} c_{0}:
\end{aligned}
$$

where $C_{n}, b_{n}$ are ghost and antighost oscillators satisfying the commutation relations

$$
\begin{aligned}
& \left\{C_{n}, b_{m}\right\}=\delta_{n,-m}, \quad\left\{C_{n}, C_{m}\right\}=0, \quad\left\{b_{n}, b_{m}\right\}=0 \\
& {\left[\gamma_{\lambda}, \beta_{\rho}\right]=\delta_{\lambda, \rho} \quad\left[\gamma_{\lambda}, \gamma_{\rho}\right]=0, \quad\left[\beta_{\lambda}, \beta_{\rho}\right]=0}
\end{aligned}
$$

$\lambda, \rho \in Z+\frac{1}{2}$ for NS superstring, and $\lambda, \rho \in Z$ for R superstring. 
From equations (8) - (10) we can derive:

$$
\begin{aligned}
Q^{2}= & \sum_{n>0}\left\{\frac{1}{8}(D-10) n^{2}+\left(g G^{2}-\frac{D}{8}+\frac{1}{4}+2 a_{0}\right)\right\} n C_{-n} C_{n} \\
& +\sum_{\lambda>0}\left\{\frac{1}{2}(D-10) \lambda^{2}+\left(g G^{2}-\frac{D}{8}+\frac{1}{4}+2 a_{0}\right)\right\} \gamma_{-\lambda} \gamma_{\lambda}
\end{aligned}
$$

in the NS-case, and

$$
\begin{aligned}
Q^{2}= & \sum_{n>0}\left\{\frac{1}{8}(D-10) n^{2}+g G^{2}+2 a_{0}\right\} n C_{-n} C_{n} \\
& +\sum_{\lambda>0}\left\{\frac{1}{2}(D-10) \lambda^{2}+g G^{2}+2 a_{0}\right\} \gamma_{-\lambda} \gamma_{\lambda}+a_{0} \gamma^{2}
\end{aligned}
$$

in the R-case.

From here it is seen that $Q^{2}=0$ when

$$
D=10, \quad a_{0}=\frac{1}{2}\left(1-g G^{2}\right)
$$

in the NS-case, and $D=10, a_{0}=0, g=0$ in the R-case

\section{EQUATIONS OF MOTION}

Let us consider the equation

$$
\left(L_{0}-a_{0}\right) \Psi[X, \psi]=0
$$

followed from the BRST equation for string field functional

$$
Q \Psi[X, \psi]=0
$$

By inserting here the explicit expression of $L_{0}$

$$
L_{0}=\frac{1}{2} \square-\sum_{k=1}^{\infty} \alpha_{-K}^{\mu} \alpha_{\mu K}-\sum_{\lambda>0} b_{-\lambda}^{\mu} b_{\mu \lambda}
$$

and using the commutation relations between the oscillators $\alpha_{n}^{\mu}$ and $b_{\lambda}^{\mu}$, we obtain the equations of motion for component fields in the expansion expression of functional $\Psi$,

$$
\begin{aligned}
& \Psi[X, \psi]=\sum_{n, s=0}^{\infty} \sum_{r ! s !}^{(-i)^{r+3} \psi_{\mu_{1} \ldots \mu_{r}, \nu_{1} \ldots \nu_{s}}^{n_{1} \ldots \nu_{s}}(x)} \\
& \text {. } \alpha_{n_{1}}^{\mu_{1}^{+}} \ldots \alpha_{n_{r}}^{\mu_{r}^{+}} b_{\lambda_{1}}^{v_{1}^{+}} \ldots . b_{\lambda_{s}}^{v_{s}^{+}}|0\rangle
\end{aligned}
$$

For example, for the component fields associated to low excited states

$$
\psi[X, \psi]=\left\{\psi(x)-i A_{v}(x) b_{\frac{1}{2}}^{v+}-i C_{\mu}(x) \alpha_{1}^{\mu+}+\ldots\right\}|0\rangle
$$


we have

$$
\begin{aligned}
& \left(\square+g G^{2}-1\right) \psi(x)=0 \\
& \left(\square+g G^{2}\right) A_{\mu}(x)=0 \\
& \left(\square+g G^{2}+1\right) C_{\mu}(x)=0
\end{aligned}
$$

Hence, the mass spectrum $M^{2}$ is shifted by an amount $g G^{2}$ as compared to conventional theory. In particular, the former tachyon field $\psi_{(x)}$ has $m^{2}=g G^{2}-1$ which is positive when $g G^{2}>1$

In general, the component field

$$
\psi_{\mu_{1} \ldots \mu_{r}, v_{1} \ldots v_{s}}^{n_{1} \ldots n_{r}, \lambda_{1} \ldots \lambda_{s}}(x)
$$

satisfies the Klein-Gordon equation

$$
\left(\square+M^{2}(n, \lambda)\right) \psi_{\mu_{1} \ldots \mu_{r}, v_{1} \ldots v_{s}}^{n_{1} \ldots n_{r}, \lambda_{1} \ldots \lambda_{s}}(x)=0
$$

with

$$
M^{2}(n, \lambda)=2\left[\sum_{j=1}^{r} n_{j}+\sum_{k=1}^{s} \lambda_{k}\right]+\left(g G^{2}-1\right) .
$$

For $R$ superstring the result remains unchanged.

\section{REFERENCES}

[1] M. B. Green, J. H. Schwarz, E. Wetten, Superstring Theory, Cambridge University Press 1987.

[2] L. Brink, M. Henneaux, Principles of String Theory, Plenum Press, New York 1988

[3] M.Kaku, Introduction to Superstring Theory, World Scientific 1989.

[4] L. Brink, D. Friedann, A. M. Polyakov, Physics and Mathematics of Strings, World Scientific 1990.

[5] Dao Vong Duc, Phuong Thi Thuy Hang, Comm.in Phys. 17 (2007) 193.

[6] F. Gliozzi, D. Olive, J. Scherk, Nucl.Phys.B 122 (1997) 253

[7] Dao Vong Duc, Basic Principles of Quantum Superstring Theory, Publishing House for Science and Technology, VAST, 2007. 